\title{
An Optimal Adaptive Variable Sample Size Scheme for the Multivariate Coefficient of Variation
}

\author{
Khai Wah Khaw ${ }^{1}$, Xin Ying Chew ${ }^{2, *}$, Ming Ha Lee ${ }^{3}$, Wai Chung Yeong ${ }^{4}$ \\ ${ }^{1}$ School of Management, Universiti Sains Malaysia, 11800 Pulau Pinang, Malaysia \\ ${ }^{2}$ School of Computer Sciences, Universiti Sains Malaysia, 11800 Pulau Pinang, Malaysia \\ ${ }^{3}$ Faculty of Engineering, Computing and Science, Swinburne University of Technology Sarawak Campus, \\ 93350 Kuching, Sarawak, Malaysia \\ ${ }^{4}$ School of Mathematical Sciences, Sunway University, 47500 Petaling Jaya, Malaysia
}

\begin{abstract}
Development of an efficient process monitoring system has always received great attention. Previous studies revealed that the coefficient of variation $(\mathrm{CV})$ is important in ensuring process quality, especially for monitoring a process where its process mean and variance are highly correlated. The fact that almost all industrial process monitoring involves a minimum of two or more related quality characteristics being monitored simultaneously, this paper incorporates the salient feature of the adaptive sample size VSS scheme into the standard multivariate CV (MCV) chart, called the VSS MCV chart. A Markov chain model is developed for the derivation of the charts performance measures, i.e the average run length (ARL), the standard deviation of the run length (SDRL), the average sample size (ASS), the average number of observations to signal (ANOS) and the expected average run length (EARL). The numerical comparison shows that the proposed chart prevails over the existing standard MCV chart for detecting small and moderate upward and downward MCV shifts.
\end{abstract}

Keywords Control charting technique, efficient process monitoring, Markov-chain, multivariate coefficient of variation, variable sample size.

AMS 2010 subject classifications 90B25, 62P30

DOI:10.19139/soic-2310-5070-996

\section{Introduction}

Control charting techniques are common-used techniques in the process signal detection for the purpose of improving the quality of manufacturing and service processes. Most of the control charts are used to monitor the process mean and variance, subject to the process mean is constant while the process standard deviation is independent of the mean. In the past few years, increasing attention has been paid to the application of the control charting techniques to various industries, such as healthcare, chemical, biological, agriculture, service, etc. However, the mean and standard deviation of some processes are not independent of each other, this may cause erroneous conclusions. To circumvent this drawback, the coefficient of variation $(\mathrm{CV})$ control chart is preferred to be used. The CV is very common and it is widely applied in various disciplines. For example, Babu and Sudha [7] applied the CV for reducing the speckle noise in ultrasound images. According to Karthik and Manjunath [23], the $\mathrm{CV}$ can be used to correct the unduly non-uniform distance between grid lines in noisy microarray images. Amelio [13] and Bakowshi et al. [1] presented the use of CV on sensory evaluation of virgin olive oil and injection pressure in a compression ignition engine, respectively. Papatsouma et al. [6] investigated whether the CV is a good estimator for the population CV using a discrete uniform distribution. Additionally, the CV can be implemented to

*Correspondence to: XinYing Chew (Email: xinying@usm.my). School of Computer Sciences, Universiti Sains Malaysia, 11800 Pulau Pinang, Malaysia.

ISSN 2310-5070 (online) ISSN 2311-004X (print)

Copyright (C) 2021 International Academic Press 
evaluate the global solar radiation in terms of time scale separation [20]. Wachter et al. [15] examined the sincerity of effort detection for grip strength based on the CV while Doring and Reckling [26] adjusted the CV for detecting the global trends of cereal yield stability.

In the last decade, numerous intermediate and advanced CV charts were introduced to increase the sensitivity of the standard CV chart of Kang et al. [3], in detecting small and moderate CV shifts, such as those by [16], [19], [10], [24], [11], to name a few. In the industrial setting, multivariate process monitoring is considered as an important procedure. A standard (Std) multivariate $\mathrm{CV}(\mathrm{MCV})$ chart was proposed to monitor the multivariate processes [30]. The variable sample size and sampling interval (VSSI) MCV has then surpassed the Std MCV chart [9]. Subsequently, Chew et al. [34], [33] implemented the variable parameter and run rules MCV charts. The findings revealed that the statistical performances of the proposed charts are significantly better than the Std MCV chart of Yeong et al. [30]. More recently, Khaw et al. [12] considered the synthetic MCV chart whereas Giner-Bosch et al. [27] recommended the exponentially weighted moving average MCV chart.

Psarakis [22] reviewed the development of the adaptive control charts. The variable sample size (VSS) scheme is one of the best adaptive features, especially when compared to the non-adaptive counterparts [4]. Prabhu et al. [25] and Costa [2] were the pioneers to develop a VSS $\bar{x}$ chart where the proposed chart is proved to be outperformed the Shewhart $\bar{x}$ chart. A VSS Hotellings $T^{2}$ chart was then proposed [5]. Castagliola et al. [18] and Hu et al. [32] investigated the VSS $\bar{x}$ chart with estimated parameters and measurement errors, respectively, while Teoh et al. [31] evaluated the performance of the VSS $\bar{x}$ chart in terms of the median run length and expected median run length. Kazemzadeh et al. [21] incorporated the VSS scheme into a simple linear profile monitoring. Chukhrova and Johannssen [14] suggested a VSS hypergeometric p-chart whereas Lim et al. [24] presented a VSS CV chart based on the median run length. The VSS scheme was also incorporated into the CV charts by Castagliola et al. [16] and Yeong et al. [29], respectively, where the charts performance in the latter was better than the former.

In the existing literature, the adoption of the VSS scheme into a Std MCV chart was briefly explained by Khaw et al. [9], where the main concept was adopted from the VSSI scheme, by letting the short and long sampling intervals to be set equal to the fixed sampling interval. This method cannot fully reflect the essentials of the VSS scheme. The goal of this paper is to further discuss on the VSS MCV chart, which includes (1) evaluate additional important performance measures for the VSS scheme such as the average sample size (ASS) and the average number of observations to signal (ANOS), (2) investigate the detection of small and moderate downward process $\mathrm{MCV}$ as this is vital for process improvement, and (3) consider the selected first sample as small sample size or large sample size. Note that these discussions were not been considered in the existing VSSI MCV chart of Khaw et al. [9]. In view of the importance of monitoring the MCV, coupled with the salient feature of the VSS scheme, this paper proposes a new VSS chart for monitoring the MCV. The VSS scheme is considered as a practical and flexible approach in increasing the effectiveness of the Std MCV chart, for detecting small and moderate MCV shifts. The VSS MCV chart provides the flexibility to quality practitioners in varying the sample size. As it will be shown in Section 4, the VSS MCV chart generally outperforms the existing Std MCV chart, in terms of the average run length (ARL), the standard deviation of the run length (SDRL), the expected average run length (EARL), the ASS and the ANOS criteria.

The remainder of the sections are organized as follows: Section 2 discusses the design of the two one-sided VSS MCV charts. The derivations of the formulae and algorithms to compute the ARL, SDRL, ASS, EARL and ANOS values, by adopting the Markov-chain method are also illustrated. The next section provides an optimization procedure of the proposed chart. Statistical comparisons among the VSS MCV and Std MCV charts are enumerated in Section 4. Lastly, the research findings and suggestions for future research are shown in the last section.

\section{The Two One-sided VSS MCV Charts}

Voinov and Nikulin [28] derived the population MCV statistic as $\gamma=\left(\mu^{T} \boldsymbol{\Sigma}^{-1} \mu\right)^{-\frac{1}{2}}$, where $\mu$ and $\sigma$ refer to the mean vector and covariance matrix, respectively. $\gamma$ can be estimated by the sample MCV, $\hat{\gamma}$ when $\mu$ and $\sigma$ are unspecified. Thus, $\hat{\gamma}=\left(\overline{\mathbf{x}}^{T} \mathbf{S}^{-1} \overline{\mathbf{x}}\right)^{-\frac{1}{2}}$ by replacing $\mu$ and $\sigma$ to $\overline{\mathbf{x}}$ and $S$. Here, $\overline{\mathbf{x}}$ is the sample mean vector whereas $S$ is the sample covariance matrix. The computations of $\overline{\mathrm{x}}$ and $S$ are shown as follows: 


$$
\overline{\mathbf{x}}=\frac{1}{n} \sum_{t=1}^{n} \mathbf{X}_{t}
$$

and

$$
\mathbf{S}=\frac{1}{n-1} \sum_{t=1}^{n}\left(\mathbf{X}_{t}-\overline{\mathbf{x}}\right)\left(\mathbf{X}_{t}-\overline{\mathbf{x}}\right)^{T},
$$

respectively, where $\overline{\mathrm{x}}$ and $S$ are independent of one another.

The Std MCV chart of Yeong et al. [30] uses a fixed sample size, $n_{0}$. The Std MCV chart consists of two regions, i.e. the safe region and the action region. Contrary to the Std MCV chart, the VSS MCV chart comprises three regions, which represent the safe, warning and action regions. The VSS scheme varies the sample size to improve the performance of the Std MCV chart in the detection of small and moderate MCV shifts. The sample size adopted for the proposed chart is varied between the small sample size, $n_{1}$ and the large sample size, $n_{2}$, where $n_{1}<A S S_{0}<n_{2}$. Note that $A S S_{0}\left(=n_{0}\right)$ is the in-control ASS, where $A S S_{0}$ of the VSS MCV chart is equal to the fixed sample size of the Std MCV chart, for ensuring a fair comparison. This paper suggests two one-sided VSS MCV charts, that are the upward and downward VSS MCV charts. The VSS MCV chart works as follows:

1. When the $t$-th sample MCV, $\hat{\gamma}$ falls in the safe region $(\hat{\gamma}$ falls below the warning limit for the upward chart, while $\hat{\gamma}$ falls above the warning limit for the downward chart), the process shows no indication of trouble. Hence, $n_{1}$ is taken to compute the $(t+1)$-th sample MCV, $\hat{\gamma}_{t+1}$;

2. When $\hat{\gamma}$ falls in the warning region ( $\hat{\gamma}$ falls above the warning limit and below the control limit for the upward chart, while $\hat{\gamma}$ falls below the warning limit and above the control limit for the downward chart), the process still shows no indication of trouble but there is a higher tendency for it to become out-of-control. Hence, $n_{2}$ is taken to compute $\hat{\gamma}_{t+1}$; and

3. When $\hat{\gamma}$ falls in action region ( $\hat{\gamma}$ falls above the control limit for the upward chart, while $\hat{\gamma}$ falls below the control limit for the downward chart), the process shows an indication of trouble at the $t$-th sample due to the presence of assignable causes. In this case, an immediate investigation should be taken.

The upper control and warning limits of the upward VSS MCV chart can be obtained as:

$$
U C L=F_{\hat{\gamma}}^{-1}\left(1-\alpha \mid n_{a}, p, \delta_{0}\right)
$$

and

$$
L C L=F_{\hat{\gamma}}^{-1}\left(\alpha \mid n_{a}, p, \delta_{0}\right)
$$

respectively, whereas the lower control and warning limits of the downward VSS MCV chart can be obtained as:

$$
U W L=F_{\hat{\gamma}}^{-1}\left(1-\alpha^{\prime} \mid n_{a}, p, \delta_{0}\right)
$$

and

$$
L W L=F_{\hat{\gamma}}^{-1}\left(\alpha^{\prime} \mid n_{a}, p, \delta_{0}\right)
$$

respectively, where $\delta_{0}=n / \gamma_{0}^{2}$ and the $\alpha^{\prime}$ value is determined based on desired in-control ARL ( $\left.A R L_{0}\right)$ value when the process MCV is in-control and $\alpha^{\prime}>\alpha$. Here, $a=1$ when $n(i)=n_{1}$ and $2=2$ when $n(i)=n_{2}$. The n(i) denotes the selected sample size. Equations (3) C (6) follows an inverse cumulative distribution function (cdf) of $\hat{\gamma}$, i.e. $F_{\hat{\gamma}}^{-1}(\alpha \mid n, p, \delta)=\sqrt{[(n(n-p)) /((n-1) p)] \times\left[1 / F_{F}^{-1}(1-\alpha \mid p, n-p, \delta)\right]}$. Here, $F_{F}^{-1}(\cdot)$ refers to an inverse cdf of a noncentral $F$ distribution while $p$ denotes the number of quality characteristics, where this distribution is only valid when $p_{i}$ n due to the positive degree of freedom and $\delta$ is defined as $n /\left(\tau \gamma_{0}\right)^{2}$, where the shift size $\tau=1$ when the process shows no indication of trouble, while $\gamma_{1}=\tau \gamma_{0}$ is an out-of-control MCV when $\tau \neq 1$. When $\tau \neq 1$, the values of $\tau>1$ and $0<\tau<1$ refer to upward and downward MCV shifts, respectively. A three-state 
Table 1. Optimal parameter combinations $\left(n_{1}, n_{2}, \alpha^{\prime}\right)$ of the VSS MCV chart for minimizing $A R L_{1}$, and $E A R L_{1}$, when $p=$ $2, A S S_{0} \in(5,10), \gamma_{0} \in(0.1,0.3,0.5), \tau \in(0.5,0.6,0.7,0.8,0.9,1.1,1.2,1.3,1.4,1.5)$ and $\left(\tau_{\min }, \tau_{\max }\right) \in([0.5,1),(1,2])$



Markov-chain model is adopted from Yeong et al. [29] to derive the formulae for the ARL and EARL of the VSS MCV chart, where $1^{\text {st }}, 2^{\text {nd }}$ and $3^{\text {rd }}$ states correspond to safe, warning and action regions, respectively. Here, the first two are transient and the last one is the absorbing state. Subsequently, the resulting transition probability matrix A can be obtained as

$$
\mathbf{A}=\left(\begin{array}{ccc}
A_{11} & A_{12} & 1-\left(A_{11}+A_{12}\right) \\
A_{21} & A_{22} & 1-\left(A_{21}+A_{22}\right) \\
0 & 0 & 1
\end{array}\right)
$$

where

$$
A_{11}=\operatorname{Pr}\left(\hat{\gamma} \leq U W L \mid n_{1}, p, \delta_{1}\right)=F_{\hat{\gamma}}\left(U W L \mid n_{1}, p, \delta_{1}\right),
$$


Table 2. Optimal parameter combinations $\left(n_{1}, n_{2}, \alpha^{\prime}\right)$ of the VSS MCV chart for minimizing $A R L_{1}$ and $E A R L_{1}$, when $p=$ $3, A S S_{0} \in(5,10), \gamma_{0} \in(0.1,0.3,0.5), \tau \in(0.5,0.6,0.7,0.8,0.9,1.1,1.2,1.3,1.4,1.5)$ and $\left(\tau_{\min }, \tau_{\max }\right) \in([0.5,1),(1,2])$

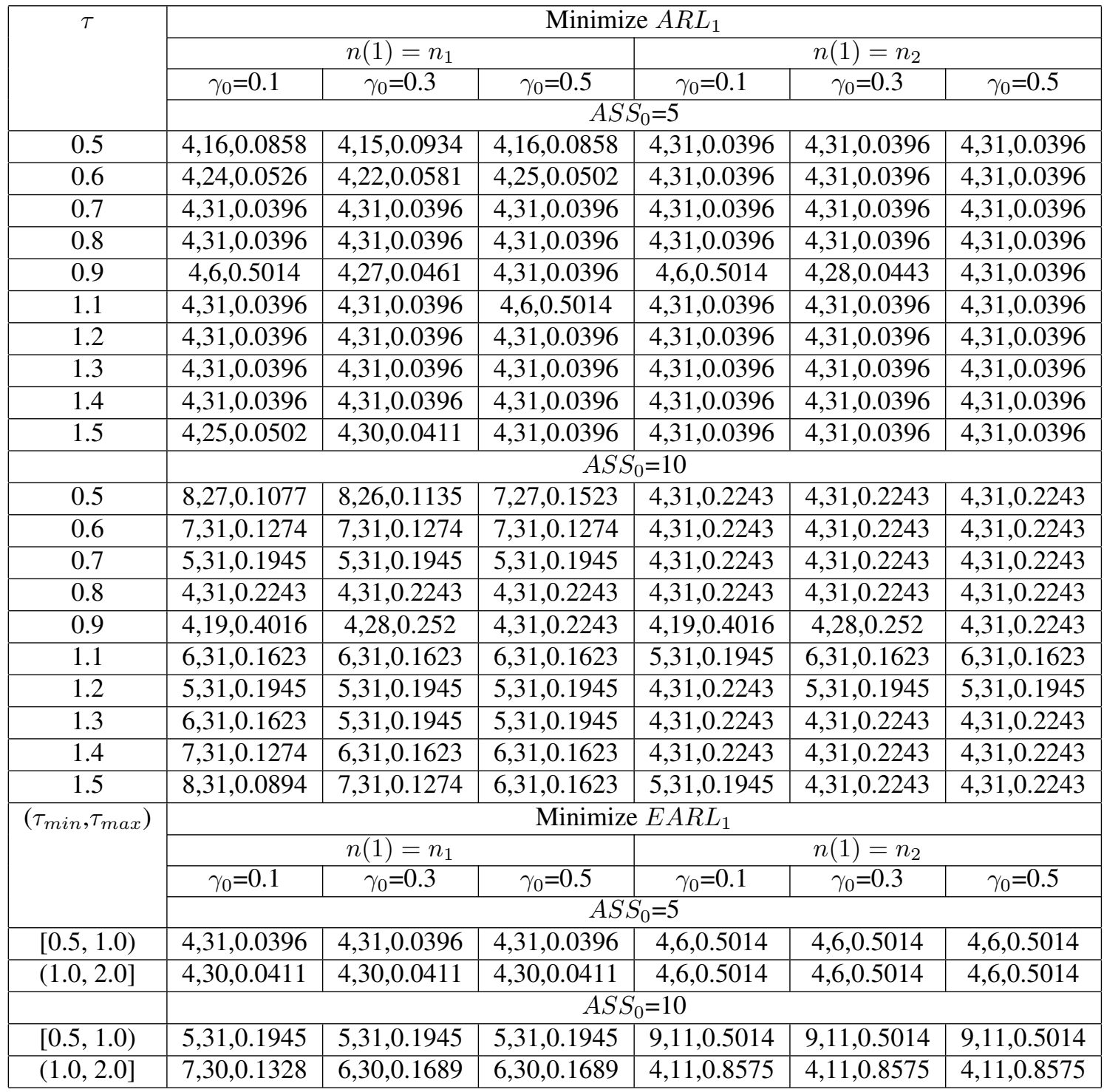

$$
\begin{gathered}
A_{12}=\operatorname{Pr}\left(U W L<\hat{\gamma} \leq U C L \mid n_{1}, p, \delta_{1}\right)=F_{\hat{\gamma}}\left(U C L \mid n_{1}, p, \delta_{1}\right)-F_{\hat{\gamma}}\left(U W L \mid n_{1}, p, \delta_{1}\right), \\
A_{21}=\operatorname{Pr}\left(\hat{\gamma} \leq U W L \mid n_{2}, p, \delta_{1}\right)=F_{\hat{\gamma}}\left(U W L \mid n_{2}, p, \delta_{1}\right), \\
A_{22}=\operatorname{Pr}\left(U W L<\hat{\gamma} \leq U C L \mid n_{2}, p, \delta_{1}\right)=F_{\hat{\gamma}}\left(U C L \mid n_{2}, p, \delta_{1}\right)-F_{\hat{\gamma}}\left(U W L \mid n_{2}, p, \delta_{1}\right),
\end{gathered}
$$

for the upward VSS MCV chart, while

$$
\begin{gathered}
A_{11}=\operatorname{Pr}\left(\hat{\gamma} \geq L W L \mid n_{1}, p, \delta_{1}\right)=1-F_{\hat{\gamma}}\left(L W L \mid n_{1}, p, \delta_{1}\right), \\
A_{12}=\operatorname{Pr}\left(L C L<\hat{\gamma} \leq L W L \mid n_{1}, p, \delta_{1}\right)=F_{\hat{\gamma}}\left(L W L \mid n_{1}, p, \delta_{1}\right)-F_{\hat{\gamma}}\left(L C L \mid n_{1}, p, \delta_{1}\right),
\end{gathered}
$$


Table 3. Optimal parameter combinations $\left(n_{1}, n_{2}, \alpha^{\prime}\right)$ of the VSS MCV chart for minimizing $A S S_{1}$, when $p \in(2,3)$, $A S S_{0} \in(5,10), \gamma_{0} \in(0.1,0.3,0.5)$ and $\tau \in(0.5,0.6,0.7,0.8,0.9,1.1,1.2,1.3,1.4,1.5)$

\begin{tabular}{|c|c|c|c|c|c|c|}
\hline \multirow[t]{5}{*}{$\tau$} & \multicolumn{6}{|c|}{ Minimize $A S S_{1}$} \\
\hline & \multicolumn{6}{|c|}{$p=2$} \\
\hline & \multicolumn{3}{|c|}{$n(1)=n_{1}$} & \multicolumn{3}{|c|}{$n(1)=n_{2}$} \\
\hline & $\gamma_{0}=0.1$ & $\gamma_{0}=0.3$ & $\gamma_{0}=0.5$ & $\gamma_{0}=0.1$ & $\gamma_{0}=0.3$ & $\gamma_{0}=0.5$ \\
\hline & \multicolumn{6}{|c|}{$A S S_{0}=5$} \\
\hline 0.5 & $3,6,0.6676$ & $3,6,0.6676$ & $3,6,0.6676$ & $3,6,0.6676$ & $3,6,0.6676$ & $3,6,0.6676$ \\
\hline 0.6 & $4,6,0.5014$ & $4,6,0.5014$ & $4,6,0.5014$ & $4,6,0.5014$ & $4,6,0.5014$ & $4,6,0.5014$ \\
\hline 0.7 & $4,6,0.5014$ & $4,6,0.5014$ & $4,6,0.5014$ & $4,6,0.5014$ & $4,6,0.5014$ & $4,6,0.5014$ \\
\hline 0.8 & $4,6,0.5014$ & $4,6,0.5014$ & $4,6,0.5014$ & $4,6,0.5014$ & $4,6,0.5014$ & $4,6,0.5014$ \\
\hline 0.9 & $4,6,0.5014$ & $4,6,0.5014$ & $4,6,0.5014$ & $4,6,0.5014$ & $4,6,0.5014$ & $4,6,0.5014$ \\
\hline 1.1 & $4,6,0.5014$ & $4,6,0.5014$ & $4,6,0.5014$ & $4,6,0.5014$ & $4,6,0.5014$ & $4,6,0.5014$ \\
\hline 1.2 & $3,6,0.6676$ & $3,6,0.6676$ & $4,6,0.5014$ & $3,6,0.6676$ & $3,6,0.6676$ & $4,6,0.5014$ \\
\hline 1.3 & $3,6,0.6676$ & $3,6,0.6676$ & $3,6,0.6676$ & $3,6,0.6676$ & $3,6,0.6676$ & $3,6,0.6676$ \\
\hline 1.4 & $3,6,0.6676$ & $3,6,0.6676$ & $3,6,0.6676$ & $3,6,0.6676$ & $3,6,0.6676$ & $3,6,0.6676$ \\
\hline \multirow[t]{2}{*}{1.5} & $3,6,0.6676$ & $3,6,0.6676$ & $3,6,0.6676$ & $3,6,0.6676$ & $3,6,0.6676$ & $3,6,0.6676$ \\
\hline & \multicolumn{6}{|c|}{$A S S_{0}=10$} \\
\hline 0.5 & $3,11,0.8753$ & $3,11,0.8753$ & $3,11,0.8753$ & $3,11,0.8753$ & $3,11,0.8753$ & $3,11,0.8753$ \\
\hline 0.6 & $3,11,0.8753$ & $3,11,0.8753$ & $3,11,0.8753$ & $3,11,0.8753$ & $3,11,0.8753$ & $3,11,0.8753$ \\
\hline 0.7 & $3,11,0.8753$ & $3,11,0.8753$ & $3,11,0.8753$ & $3,11,0.8753$ & $3,11,0.8753$ & $3,11,0.8753$ \\
\hline 0.8 & $9,11,0.5014$ & $9,11,0.5014$ & $9,11,0.5014$ & $9,11,0.5014$ & $9,11,0.5014$ & $9,11,0.5014$ \\
\hline 0.9 & $9,11,0.5014$ & $9,11,0.5014$ & $9,11,0.5014$ & $9,11,0.5014$ & $9,11,0.5014$ & $9,11,0.5014$ \\
\hline 1.1 & $3,11,0.8753$ & $3,11,0.8753$ & $3,11,0.8753$ & $3,11,0.8753$ & $3,11,0.8753$ & $3,11,0.8753$ \\
\hline 1.2 & $3,11,0.8753$ & $3,11,0.8753$ & $3,11,0.8753$ & $3,11,0.8753$ & $3,11,0.8753$ & $3,11,0.8753$ \\
\hline 1.3 & $3,11,0.8753$ & $3,11,0.8753$ & $3,11,0.8753$ & $3,11,0.8753$ & $3,11,0.8753$ & $3,11,0.8753$ \\
\hline 1.4 & $3,11,0.8753$ & $3,11,0.8753$ & $3,11,0.8753$ & $3,11,0.8753$ & $3,11,0.8753$ & $3,11,0.8753$ \\
\hline 1.5 & $3,11,0.8753$ & $3,11,0.8753$ & $3,11,0.8753$ & $3,11,0.8753$ & $3,11,0.8753$ & $3,11,0.8753$ \\
\hline \multirow[t]{4}{*}{$\tau$} & \multicolumn{6}{|c|}{$p=3$} \\
\hline & \multicolumn{3}{|c|}{$n(1)=n_{1}$} & \multicolumn{3}{|c|}{$n(1)=n_{2}$} \\
\hline & $\gamma_{0}=0.1$ & $\gamma_{0}=0.3$ & 5 & $-\Omega$ & $\gamma_{0}=0.3$ & $\gamma_{0}=0.5$ \\
\hline & \\
\hline 0.5 & $4,31,0.0396$ & 4,31, & $4,31,0.0396$ & $4,31,0.0396$ & $4,31,0.0396$ & $4,31,0.0396$ \\
\hline 0.6 & $4,6,0.5014$ & $4,6,0.5014$ & $4,6,0.5014$ & $4,6,0.5014$ & $4,6,0.5014$ & $4,6,0.5014$ \\
\hline 0.7 & $4,6,0.5014$ & 014 & $4,6,0.5014$ & $4,6,0.5014$ & 5014 & $4,6,0.5014$ \\
\hline 0.8 & $4,6,0.5014$ & $4,6,0.5014$ & $4,6,0.5014$ & $4,6,0.5014$ & $4,6,0.5014$ & $4,6,0.5014$ \\
\hline 0.9 & $4,6,0.5014$ & $4,6,0.5014$ & $4,6,0.5014$ & $4,6,0.5014$ & $4,6,0.5014$ & $4,6,0.5014$ \\
\hline 1.1 & $4,6,0.5014$ & $4,6,0$ & $4,6,0.5014$ & $4,6,0.5014$ & 5014 & $4,6,0.5014$ \\
\hline 1.2 & $4,6,0.5014$ & $4,6,0.5014$ & $4,6,0.5014$ & $4,6,0.5014$ & $4,6,0.5014$ & $4,6,0.5014$ \\
\hline 1.3 & $4,6,0.5014$ & 014 & $4,6,0.5014$ & $4,6,0.5014$ & 014 & $4,6,0.5014$ \\
\hline 1.4 & $4,6,0.5014$ & 4,6, & $4,6,0.5014$ & 014 & 4,6 & $4,6,0.5014$ \\
\hline 1.5 & $4,6,0.5014$ & $4,6,0.5014$ & $4,6,0.5014$ & $4,6,0.5014$ & $4,6,0.5014$ & $4,6,0.5014$ \\
\hline & \multicolumn{6}{|c|}{$A S S_{0}=10$} \\
\hline 0.5 & $4,11,0.8575$ & $4,11,0.8575$ & $4,11,0.8575$ & $4,11,0.8575$ & $4,11,0.8575$ & $4,11,0.8575$ \\
\hline 0.6 & $4,11,0.8575$ & $4,11,0.8575$ & $4,11,0.8575$ & $4,11,0.8575$ & $4,11,0.8575$ & $4,11,0.8575$ \\
\hline 0.7 & $4,11,0.8575$ & $4,11,0.8575$ & $4,11,0.8575$ & $4,11,0.8575$ & $4,11,0.8575$ & $4,11,0.8575$ \\
\hline 0.8 & $9,11,0$ & $9,11,($ & $9,11,0.5014$ & $9,11,0.5014$ & $9,11,0.5014$ & $9,11,0.5014$ \\
\hline 0.9 & $9,11,0.5014$ & $9,11,0.5014$ & $9,11,0.5014$ & $9,11,0.5014$ & $9,11,0.5014$ & $9,11,0.5014$ \\
\hline 1.1 & $4,11,0.8575$ & $4,11,0.8575$ & $9,11,0.5014$ & $4,11,0.8575$ & $4,11,0.8575$ & $9,11,0.5014$ \\
\hline 1.2 & $4,11,0.8575$ & $4,11,0.8575$ & $4,11,0.8575$ & $4,11,0.8575$ & $4,11,0.8575$ & $4,11,0.8575$ \\
\hline 1.3 & $4,11,0.8575$ & $4,11,0.8575$ & $4,11,0.8575$ & $4,11,0.8575$ & $4,11,0.8575$ & $4,11,0.8575$ \\
\hline 1.4 & $4,11,0.8575$ & $4,11,0.8575$ & $4,11,0.8575$ & $4,11,0.8575$ & $4,11,0.8575$ & $4,11,0.8575$ \\
\hline 1.5 & $4,11,0.8575$ & $4,11,0.8575$ & $4,11,0.8575$ & $4,11,0.8575$ & $4,11,0.8575$ & $4,11,0.8575$ \\
\hline
\end{tabular}

$$
A_{21}=\operatorname{Pr}\left(\hat{\gamma} \geq L W L \mid n_{2}, p, \delta_{1}\right)=1-F_{\hat{\gamma}}\left(L W L \mid n_{2}, p, \delta_{1}\right),
$$


Table 4. $A R L_{1}, S D R L_{1}$ and $E A R L_{1}$ of the Std MCV chart, when $p \in(2,3), A S S_{0} \in(5,10), n(1)=n_{1}, \gamma_{0} \in$ $(0.1,0.3,0.5), \tau \in(0.5,0.6,0.7,0.8,0.9,1.1,1.2,1.3,1.4,1.5)$ and $\left(\tau_{\min }, \tau_{\max }\right) \in([0.5,1),(1,2])$

\begin{tabular}{|c|c|c|c|c|c|c|}
\hline \multirow[t]{4}{*}{$\tau$} & \multicolumn{6}{|c|}{$A R L_{1}, S D R L_{1}$} \\
\hline & \multicolumn{3}{|c|}{$p=2$} & \multicolumn{3}{|c|}{$p=3$} \\
\hline & $\gamma_{0}=0.1$ & $\gamma_{0}=0.3$ & $\gamma_{0}=0.5$ & $\gamma_{0}=0.1$ & $\gamma_{0}=0.3$ & $\gamma_{0}=0.5$ \\
\hline & \multicolumn{6}{|c|}{$A S S_{0}=5$} \\
\hline 0.5 & $56.43,55.93$ & $52.15,51.65$ & $56.37,55.87$ & $102.21,101.71$ & $97.27,96.77$ & $103.33,102.83$ \\
\hline 0.6 & $94.03,93.53$ & $87.72,87.22$ & $93.57,93.07$ & $145.17,144.67$ & $139.23,138.73$ & $146.12,145.62$ \\
\hline 0.7 & $143.67,143.17$ & $135.3,134.8$ & $142.82,142.31$ & $193.46,192.96$ & $186.39,185.89$ & $194.52,194.02$ \\
\hline 0.8 & $206.98,206.48$ & $198.12,197.62$ & $204.62,204.12$ & $248.48,247.98$ & $242.14,241.64$ & $247.78,247.28$ \\
\hline 0.9 & $281.24,280.74$ & $277.14,276.64$ & $282.74,282.24$ & $305.76,305.26$ & $304.71,304.21$ & $309.54,309.04$ \\
\hline 1.1 & $118.63,118.13$ & $125.71,125.21$ & $142.99,142.49$ & $133.52,133.02$ & $140.67,140.17$ & $158.14,157.64$ \\
\hline 1.2 & $50.45,49.95$ & $55.77,55.27$ & $69.68,69.18$ & $61.45,60.95$ & $67.36,66.86$ & $82.78,82.28$ \\
\hline 1.3 & $26.16,25.65$ & $29.85,29.34$ & $39.97,39.46$ & $33.59,33.09$ & $37.98,37.47$ & $50.02,49.52$ \\
\hline 1.4 & $15.64,15.14$ & $18.28,17.77$ & $25.78,25.28$ & $20.8,20.3$ & $24.1,23.6$ & $33.54,33.04$ \\
\hline \multirow[t]{2}{*}{1.5} & $10.39,9.88$ & $12.37,11.86$ & $18.14,17.63$ & $14.13,13.62$ & $16.7,16.19$ & $24.3,23.79$ \\
\hline & \multicolumn{6}{|c|}{$A S S_{0}=10$} \\
\hline 0.5 & $6.59,6.07$ & $6.14,5.62$ & $7.21,6.69$ & $9.22,8.71$ & $8.46,7.95$ & $9.88,9.37$ \\
\hline 0.6 & $17.5,16.99$ & $15.94,15.44$ & $18.55,18.05$ & $23.33,22.83$ & $21.05,20.55$ & $24.27,23.76$ \\
\hline 0.7 & $43.56,43.05$ & $39.28,38.77$ & $44.45,43.94$ & $54.02,53.52$ & $48.6,48.1$ & $54.48,53.97$ \\
\hline 0.8 & $99.02,98.52$ & $89.55,89.05$ & $97.66,97.16$ & $113.49,112.99$ & $103.03,102.53$ & $111.62,111.12$ \\
\hline 0.9 & $200.53,200.03$ & $190.22,189.72$ & $197.42,196.92$ & $213.16,212.66$ & $203.7,203.2$ & $210.64,210.14$ \\
\hline 1.1 & $81.09,80.59$ & $88.92,88.42$ & $105.06,104.56$ & $86.27,85.77$ & $94.3,93.8$ & $110.93,110.43$ \\
\hline 1.2 & $27.3,26.79$ & $31.77,31.27$ & $41.83,41.32$ & $30.1,29.59$ & $34.9,34.4$ & $45.74,45.23$ \\
\hline 1.3 & $12.33,11.82$ & $14.91,14.4$ & $21.01,20.5$ & $13.86,13.35$ & $16.7,16.2$ & $23.49,22.98$ \\
\hline 1.4 & $6.85,6.33$ & $8.46,7.95$ & $12.42,11.91$ & $7.76,7.25$ & $9.58,9.07$ & $14.09,13.58$ \\
\hline 1.5 & $4.41,3.88$ & $5.51,4.98$ & $8.27,7.75$ & $5.01,4.48$ & $6.26,5.74$ & $9.45,8.94$ \\
\hline \multirow[t]{4}{*}{$\left(\tau_{\min }, \tau_{\max }\right)$} & \multicolumn{6}{|c|}{$E A R L_{1}$} \\
\hline & \multicolumn{3}{|c|}{$p=2$} & \multicolumn{3}{|c|}{$p=3$} \\
\hline & $\gamma_{0}=0.1$ & $\gamma_{0}=0.3$ & $\gamma_{0}=0.5$ & $\gamma_{0}=0.1$ & $\gamma_{0}=0.3$ & $\gamma_{0}=0.5$ \\
\hline & \multicolumn{6}{|c|}{$A S S_{0}=5$} \\
\hline$[0.5,1.0)$ & 180.24 & 185.92 & 186.14 & 220.18 & 224.01 & 226.33 \\
\hline \multirow[t]{2}{*}{$(1.0,2.0]$} & 39.08 & 41.85 & 49.29 & 44.59 & 47.81 & 56.62 \\
\hline & \multicolumn{6}{|c|}{$A S S_{0}=10$} \\
\hline$[0.5,1.0)$ & 100.21 & 105.57 & 106.20 & 109.10 & 114.82 & 115.53 \\
\hline$(1.0,2.0]$ & 27.60 & 29.90 & 34.97 & 29.02 & 31.46 & 36.94 \\
\hline
\end{tabular}

$$
A_{22}=\operatorname{Pr}\left(L C L<\hat{\gamma} \leq L W L \mid n_{2}, p, \delta_{1}\right)=F_{\hat{\gamma}}\left(L W L \mid n_{2}, p, \delta_{1}\right)-F_{\hat{\gamma}}\left(L C L \mid n_{2}, p, \delta_{1}\right),
$$

for the downward VSS MCV chart. Equations (8) to (15) follow the cdf of $\hat{\gamma}$, i.e. $F_{\hat{\gamma}}(x \mid n, p, \delta)=1-F_{F}[(n(n-$ $\left.p)) /\left((n-1) p x^{2}\right) \mid p, n-p, \delta\right]$, where $F_{F}(\cdot)$ denotes the cdf of a non-central F distribution, and $\delta_{1}=n_{a} / \gamma_{1}^{2}$, where $a=1$ (corresponds to small sample size) and 2 (corresponds to large sample size).

Subsequently, the out-of-control ARL $\left(A R L_{1}\right)$ and the out-of-control SDRL $\left(S D R L_{1}\right)$ of the VSS MCV chart are obtained as

$$
A R L_{1}=\mathbf{s}^{T}(\mathbf{I}-\mathbf{Q})^{-1} \mathbf{1}
$$

and

$$
S D R L_{1}=\sqrt{2 \mathbf{s}^{T}(\mathbf{I}-\mathbf{Q})^{-2} \mathbf{Q} \mathbf{1}-A R L^{2}+A R L},
$$


respectively, where

$\mathbf{s}=2 \times 1$ vector of starting probabilities such that $\mathbf{s}=(1,0)^{T}$ and $\mathbf{s}=(0,1)^{T}$ with first sample size $n_{1}$ and $n_{2}$, respectively.

$\mathbf{I}=2 \times 2$ identity matrix

$\mathbf{Q}=2 \times 2$ transition probability matrix associated with the transient states

$\mathbf{1}=(1,1)^{T}$

The out-of-control ASS $\left(A S S_{1}\right)$ denotes the ratio between the expected total number of observations taken in an infinite production horizon, which can be computed as [29]

$$
A S S_{1}=\left(n_{1}, n_{2}, n(1)\right) \theta,
$$

where $\theta$ can be obtained from $\theta=\mathbf{B}^{-1}(\mathbf{s}, 0)^{T}$. Consequently, the matrix $\mathbf{B}$ shows

$$
\mathbf{B}=\left(\begin{array}{ccc}
1 & 1 & 1 \\
A_{12} & A_{22}-1 & 0 \\
1-A_{11}-A_{12} & 1-A_{21}-A_{22} & -1
\end{array}\right),
$$

for $n(1)=n_{1}$, when the first sample size is considered as $n_{1}$, while

$$
\mathbf{B}=\left(\begin{array}{ccc}
A_{11}-1 & A_{21} & 0 \\
1 & 1 & 1 \\
1-A_{11}-A_{12} & 1-A_{21}-A_{22} & -1
\end{array}\right),
$$

for $n(1)=n_{2}$, when the first sample size is considered as $n_{2}$. It is worth noting that the performance evaluation of the VSS-type chart does not solely depend on ARL since the actual number of observations taken in a sample could not be ignored. In this case, it is important to use the out-of-control ANOS $\left(A N O S_{1}\right)$ as one of the performance measures, where the equation shows as follows:

$$
A N O S_{1}=A R L_{1} \times A S S_{1} .
$$

Practitioners need to determine $\tau$ for the computation of the ARL. However, this could be a challenge in real industry applications since $\tau$ is not easily specified. The EARL can be applied to evaluate the charts performance. Here, the in-control EARL ( $\left.E A R L_{0}\right)$ value of the VSS MCV charts is set equal to the $A R L_{0}$ value, whereas the $E A R L_{1}$ formula can be written as

$$
E A R L_{1}=\int_{\tau_{\min }}^{\tau_{\max }} A R L_{1} \times f_{\tau}(\tau) d \tau
$$

where $f_{\tau}(\tau)$ denotes the probability density function (pdf) of $\tau$. Additionally, $\tau_{\min }$ is the lower bound while $\tau_{\max }$ is the upper bound for $\tau$. It is often hard to fit the actual shape of $f_{\tau}(\tau)$ due to a lack of information on $f_{\tau}(\tau)$. To circumvent this, uniform distribution can be used. According to Siddall [8], a uniform distribution is one of the best options if practitioners are unable to specify the random variable distribution, and can only specify its upper and lower bounds. Castagliola et al. [17] suggested the interval $\left(\tau_{\min }, \tau_{\max }\right)$ for the downward and upward $E W M A C V^{2}$ charts as $=[0.5,1)$ and $(1,2]$, respectively.

\section{Optimization Algorithm}

This section presents an optimization procedure to obtain the optimal parameters of the VSS MCV chart to minimize the $A R L_{1}$ and $A S S_{1}$. It is assumed that both $A R L_{0}$ and $E A R L_{0}$ are 370. Three optimization criteria are considered to compute the optimal parameter combinations $\left(n_{1}, n_{2}, \alpha^{\prime}\right)$ for minimizing the following objective function: 
- $\operatorname{Min}_{\left(n_{1}, n_{2}, \alpha^{\prime}\right)} A R L_{1}(\tau)$, subject to $A R L_{0}$ and $A S S_{0}$

- $\operatorname{Min}_{\left(n_{1}, n_{2}, \alpha^{\prime}\right)} A S S_{1}(\tau)$, subject to $A R L_{0}$ and $A S S_{0}$

- $\operatorname{Min}_{\left(n_{1}, n_{2}, \alpha^{\prime}\right)} E A R L_{1}(\tau)$, subject to $E A R L_{0}$ and $A S S_{0}$

The optimization procedure of the VSS MCV chart is employed, i.e.

1. Determine $A R L_{0}$ (or $E A R L_{0}$ ), $A S S_{0}, p$ and $\tau$ (for minimizing $A R L_{1}(\tau)$ and $A S S_{1}(\tau)$ ), or $\left(\tau_{\min }, \tau_{\max }\right.$ ) (for minimizing $E A R L_{1}\left(\tau_{\min }, \tau_{\max }\right)$ ).

2. Start with $n_{1}=p+1$, followed by $n_{2}=A S S_{0}+1$, where $n_{1}>p$.

3. Compute $\alpha^{\prime}$ from Equation (23) subject to $A R L_{0}$ (or $E A R L_{0}$ ) $=370$ and $A S S_{0}=n_{0}$, which is adopted from Khaw et al. [9].

$$
\alpha^{\prime}=1-\frac{F_{\hat{\gamma}}\left(U C L \mid A S S_{0}, p, \delta_{0}\right)\left(A S S_{0}-n_{2}\right)}{n_{1}-n_{2}}
$$

4. Compute $A R L_{1}(\tau), S D R L_{1}(\tau), A S S_{1}(\tau), A N O S_{1}(\tau)$ and $E A R L_{1}\left(\tau_{\min }, \tau_{\max }\right)$ from Equations (16), (17), (18), (21) and (22), respectively, from the parameter combination $\left(n_{1}, n_{2}, \alpha^{\prime}\right)$.

5. Increase $n_{1}$ by 1 while maintaining the value $n_{2}$.

6. Repeat Steps 3 C 5 until $n_{1}=A S S_{0}-1$.

7. Reset $n_{1}=p+1$ and increase $n_{2}$ by 1 .

8. Repeat Steps 3 C 7 until $n_{2}=31$. According to Castagliola et al. [16], extremely large sample sizes are unlikely to be used in the industrial context. Here, the maximum sample size of 31 set in this paper only serves as guide, since the actual number of sample size taken depends on the specific process.

9. Select the optimal parameter combination $\left(n_{1}, n_{2}, \alpha^{\prime}\right)$ which minimizes the $A R L_{1}(\tau)$ (for objective function (i)), the $A S S_{1}(\tau)$ (for objective function (ii)) and the $E A R L_{1}(\tau)$ (for objective function (iii)).

\section{Results and Discussion}

The performance of the Std MCV and the proposed VSS MCV charts will be compared in this section. The Std MCV chart has a fixed sample size, $n_{0}$. For ensuring a fair comparison with the Std MCV chart in terms of the ARL and EARL criteria, the $A S S_{0}$ of the VSS MCV chart is set as $n_{0}$. In this paper, $A S S_{0}\left(=n_{0}\right) \in(5,10)$ are considered. According to Castagliola et al. [16], an extremely large $n_{2}$ value is not practical in the industry. Thus, the maximum $n_{2}$ value is set as 31 to minimize the $A R L_{1}, A S S_{1}$ and $E A R L_{1}$ values, for detecting both the increase and decrease of MCV shifts. In addition, $\tau \in(0.5,0.6,0.7,0.8,0.9,1.1,1.2,1.3,1.4,1.5),\left(\tau_{\min }, \tau_{\max }\right) \in$ ([0.5,1), $(1,2]), p \in(2,3), A S S_{0} \in(5,10)$ and $\gamma_{0} \in(0.1,0.3,0.5)$ are considered, with $n(1)=n_{1}$. Subsequently, $A R L_{0}$ is assumed to be 370. Due to space constraint, the $A R L_{1}, A S S_{1}, S D R L_{1}, A N O S_{1}$ and $E A R L_{1}$ values of the VSS MCV charts with $n(1)=n_{2}$ are not presented here. However, these values can be obtained from the first author, upon request. Tables $1 \mathrm{C} 3$ present the optimal parameter combinations $\left(n_{1}, n_{2}, \alpha^{\prime}\right)$ of the VSS MCV chart for minimizing the $A R L_{1}, A S S_{1}$ and $E A R L_{1}$ values, for detecting both the downward and upward MCV shifts, when $\tau \in(0.5,0.6,0.7,0.8,0.9,1.1,1.2,1.3,1.4,1.5),\left(\tau_{\min }, \tau_{\max }\right) \in([0.5,1),(1,2]), p \in(2,3), A S S_{0} \in(5,10)$ and $\gamma_{0} \in(0.1,0.3,0.5)$. For example, from Table 1 , when $p=2, A S S_{0}=10, \gamma_{0}=0.3, n(1)=n_{1}$ and $\tau=1.2$, the optimal parameter combination $\left(n_{1}, n_{2}, \alpha^{\prime}\right)$ of the VSS MCV chart to minimize the $A R L_{1}$ value is $(4,31,0.2243)$.

Tables $4 \mathrm{C} 6$ show the $A R L_{1}, A S S_{1}, S D R L_{1}, A N O S_{1}$ and $E A R L_{1}$ values of the VSS MCV chart, together with the $A R L_{1}, S D R L_{1}$ and $E A R L_{1}$ values of the Std MCV chart [30], for detecting both the upward and downward MCV shifts. The results show that the VSS MCV chart outperforms the Std MCV chart, for detecting both the small and moderate upward and downward MCV shifts, in terms of the $A R L_{1}, S D R L_{1}$ and $E A R L_{1}$ criteria. For example, from Table 6, when $p=3, A S S_{0}=5, \gamma_{0}=0.1$ and $\tau=1.4$, the VSS MCV chart shows the $A R L_{1}=10.69$ (for minimizing $A R L_{1}$ ) and $A R L_{1}=18.95$ (for minimizing $A S S_{1}$ ), while the Std MCV chart gives an $A R L_{1}$ of 20.8. For another example from Table 5, when $p=2, A S S_{0}=10, \gamma_{0}=0.5$ and $\left(\tau_{\min }, \tau_{\max }\right)=(1,2]$, the VSS MCV and Std MCV charts give $E A R L_{1}=30.39$ and 34.97, respectively. These results show that the VSS MCV chart has smaller $A R L_{1}, S D R L_{1}$ and $E A R L_{1}$ values than the Std MCV chart in detecting both the upward 


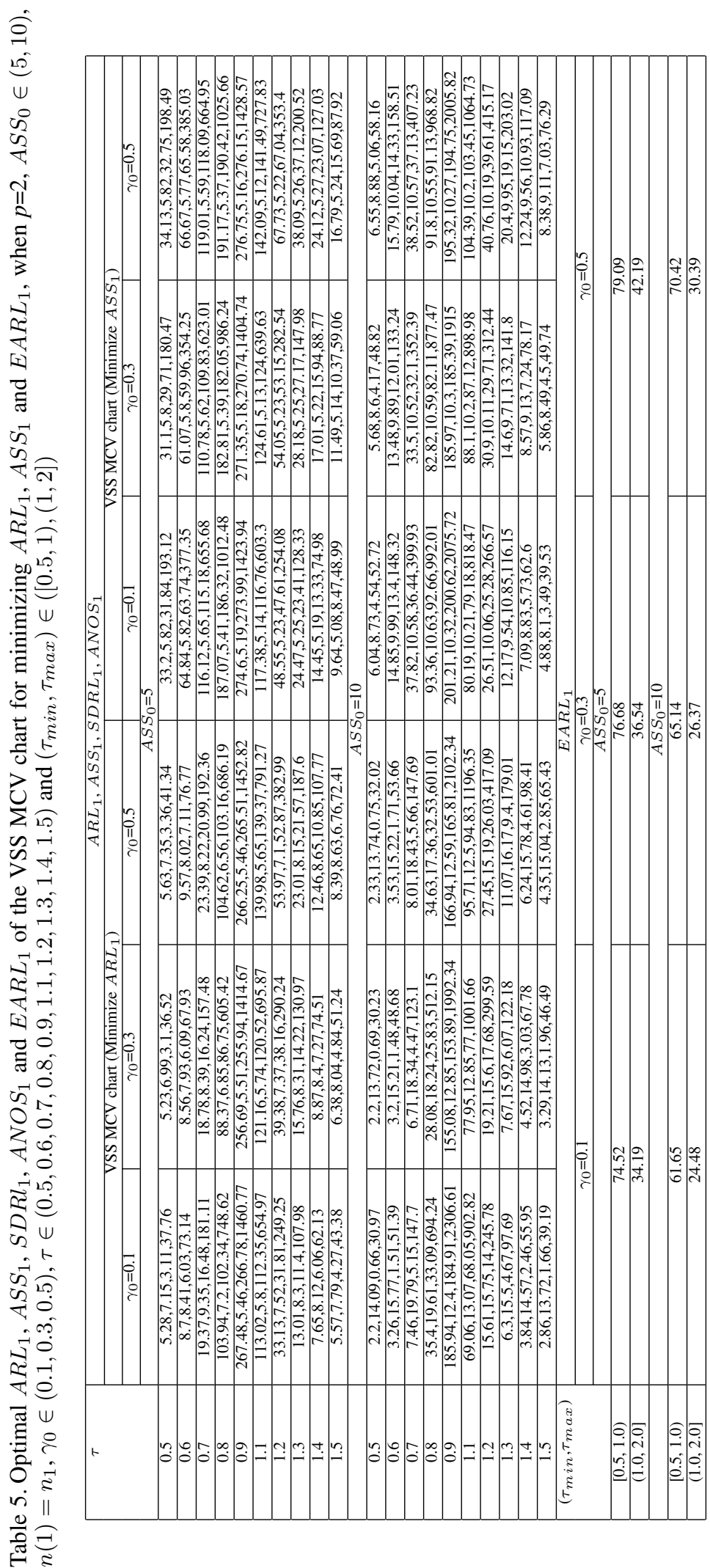




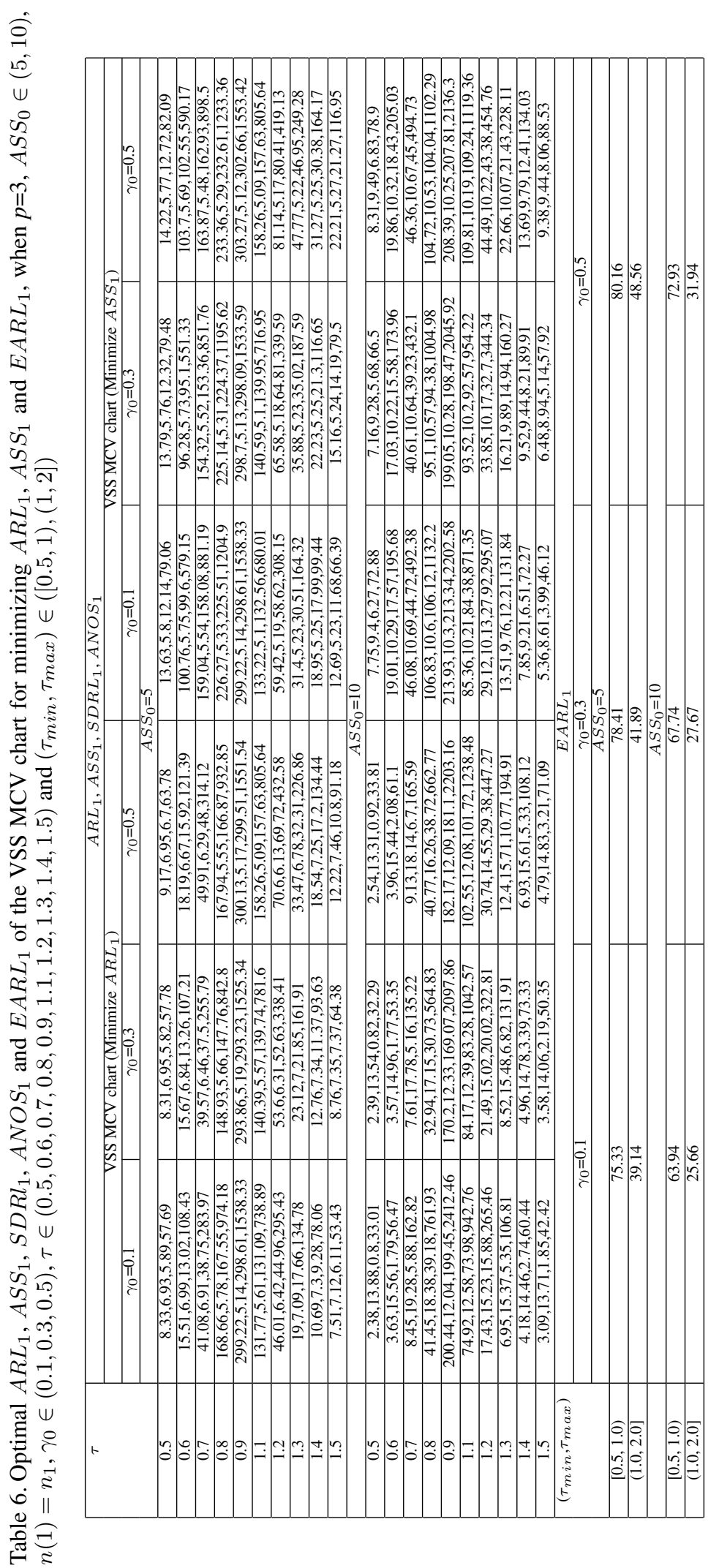

Stat., Optim. Inf. Comput. Vol. 9, September 2021 
and downward MCV shifts. The $A N O S_{1}$ value is presented in Tables $5 \mathrm{C} 6$. The results show a decreasing trend from small to large MCV shifts. For example, from Table 5, when when $p=2, A S S_{0}=5, \gamma_{0}=0.1$ and $\tau$ increases from 0.5 to 0.9 , the $A N O S_{1}$ values of the VSS MCV chart increases from 37.76 to 1460.70 (for minimizing $A R L_{1}$ ), and increases from 193.12 to 1423.94 (for minimizing $A S S_{1}$ ). Note that the $\tau$ which is closer to one indicates a smaller MCV shift. Thus, $\tau=0.5$ is a larger MCV shift than $\tau=0.9$.

\section{Conclusion}

Two one-sided VSS MCV charts are developed in this study to monitor the MCV, in terms of the $A R L_{1}$, $A S S_{1}, S D R L_{1}, A N O S_{1}$ and $E A R L_{1}$ criteria. The two one-sided charts can circumvent the biased statistical performances of their two-sided counterpart. From the results, it reveals that the VSS MCV chart performs better than the Std MCV charts, for detecting both small and moderate upward and downward MCV shifts. The two one-sided VSS MCV charts have the flexibility in allowing the sample size to be varied based on the current process quality. This flexibility enhances the effectiveness of the process monitoring system and simultaneously cost saving in the production. In future research, the two one-sided VSS MCV charts can be further investigated when measurement errors exist.

\section{Acknowledgment}

This work was funded by the Ministry of Higher Education Malaysia, Fundamental Research Grant Scheme [Grant Number: 203.PMGT.6711755].

\section{REFERENCES}

1. A. Bakowski, L. Radziszewski, M. Zmindak, Optimal control of the minimal time crisis problem by non-smooth analysis, Procedia Engineering, vol. 177, pp. 297-302, 2017.

2. A.F.B. Costa, $\bar{x}$ charts with variable sample size, Journal of Quality Technology, vol. 26, pp. 155-163, 1994.

3. C.W. Kang, M.S. Lee, Y.J. Seong, D.M. Hawkins, A control chart for the coefficient of variation, Journal of Quality Technology, vol. 39, pp. 151-158, 2007.

4. E.K. Epprecht, A.F.B. Costa, F.C.T. Mendes, Adaptive control charts for attributes, IIE Transactions, vol. 35, pp. 567-582, 2003.

5. F. Aparisi, Hotellings T2 control chart with adaptive sample sizes, International Journal of Production Research, vol. 34, pp. 2853-2862, 1996.

6. I. Papatsouma, R. Mahmoudvand, N. Farmakis, Evaluating the goodness of the sample coefficient of variation via discrete uniform distribution, Statistics, Optimization and Information Computing, vol. 7, pp. 642-652, 2019.

7. J.J.J. Babu, G.F. Sudha, Adaptive speckle reduction in ultrasound images using fuzzy logic on coefficient of variation, Biomedical Signal Processing and Control, vol. 23, pp. 93-103, 2016.

8. J.N. Siddall, Probabilistic Engineering Design-Principles and Applications, Marcel Dekker, New York, 1983.

9. K.W. Khaw, M.B.C. Khoo, P. Castagliola, M.A. Rahim, New adaptive control charts for monitoring the multivariate coefficient of variation, Computers Industrial Engineering, vol. 126, pp. 595-610, 2018.

10. K.W. Khaw, M.B.C. Khoo, W.C. Yeong, Z. Wu, Monitoring the coefficient of variation using a variable sample size and sampling interval control chart, Communications in Statistics - Simulation and Computation, vol. 46, pp. 5772-5794, 2017.

11. K.W. Khaw, X.Y. Chew, A re-evaluation of the run rules control chart for monitoring the coefficient of variation, Statistics, Optimization Information Computing, vol. 7, pp. 716-730, 2019.

12. K.W. Khaw, X.Y. Chew, W.C. Yeong, S.L. Lim, Optimal design of the synthetic control chart for monitoring the multivariate coefficient of variation, Chemometrics and Intelligent Laboratory Systems, vol. 186, pp. 33-40, 2019.

13. M. Amelio, Olive oil sensory evaluation: An alternative to the robust coefficient of variation (CVr) for measuring panel group performance in official tasting sessions, Trends in Food Science Technology, vol. 88, pp. 567-570, 2019.

14. N. Chukhrova, A. Johannssen, Hypergeometric p- chart with dynamic probability control limits for monitoring processes with variable sample and population sizes, Computers Industrial Engineering, vol. 136, pp. 681-701, 2019.

15. N.J. Wachter, M. Mentzel, R. Hutz, J. Gulke, Reliability of the grip strength coefficient of variation for detecting sincerity in normal and blocked median nerve in healthy adults, Hand Surgery and Rehabilitationl, vol. 36, pp. 90-96, 2017.

16. P. Castagliola, A. Achouri, H. Taleb, G. Celano, S. Psarakis, Monitoring the coefficient of variation using a variable sample size control chart The International Journal of Advanced Manufacturing Technology, vol. 80, pp. 1561-1576, 2015.

17. P. Castagliola, G. Celano, S. Psarakis, Monitoring the coefficient of variation using EWMA charts, Journal of Quality Technology, vol. 43, pp. 249-265, 2011. 
18. P. Castagliola, Y. Zhang, A.F.B. Costa, P. Maravelakis, The variable sample size $\bar{x}$ chart with estimated parameters, Quality and Reliability Engineering International, vol. 28, pp. 687-699, 2012.

19. P.H. Tran, K.P. Tran, The efficiency of CUSUM schemes for monitoring the coefficient of variation, Applied Stochastic Models in Business and Industry, vol. 32, pp. 870-881, 2016.

20. R. Calif, T. Soubdhan, On the use of the coefficient of variation to measure spatial and temporal correlation of global solar radiation, Renewable Energy, vol. 88, pp. 192-199, 2016.

21. R.B. Kazemzadeh, A. Amiri, B. Kouhestani, Monitoring simple linear profiles using variable sample size schemes, Journal of Statistical Computation and Simulation, vol. 86, pp. 2923-2945, 2016.

22. S. Psarakis, Adaptive control charts: Recent developments and extensions, Quality and Reliability Engineering International, vol. 31, pp. 1265-1280, 2015.

23. S.A. Karthik, S.S. Manjunath, Automatic gridding of noisy microarray images based on coefficient of variation, Informatics in Medicine Unlocked, vol. 17, pp. 100264, 2019.

24. S.L. Lim, W.C. Yeong, M.B.C. Khoo, Z.L. Chong, K.W. Khaw, An alternative design for the variable sample size coefficient of variation chart based on the median run length and expected median run length, International Journal of Industrial Engineering: Theory, Applications and Practice, vol. 26, pp. 199-220, 2019.

25. S.S. Prabhu, G.C. Runger, J.B. Keats, $\bar{x}$ chart with adaptive sample sizes, International Journal of Production Research, vol. 31 , pp. 2895-2909, 1993.

26. T.F. Doring, M. Reckling, Detecting global trends of cereal yield stability by adjusting the coefficient of variation, European Journal of Agronomy, vol. 99, pp. 30-36, 2018.

27. V. Giner-Bosch, K.P. Tran, P. Castagliola, M.B.C. Khoo, An EWMA control chart for the multivariate coefficient of variation, Quality and Reliability Engineering International, vol. 35, pp. 1515-1541, 2019.

28. V.G. Voinov, M.S. Nikulin, Unbiased estimator and their applications, multivariate case (2nd edn), Kluwer, Dordrecht, 1996.

29. W.C. Yeong, M.B.C. Khoo, S.L. Lim, M.H. Lee, A direct procedure for monitoring the coefficient of variation using a variable sample size scheme, Communications in Statistics C Simulation and Computation, vol. 46, pp. 4210-4225, 2017.

30. W.C. Yeong, M.B.C. Khoo, W.L. Teoh, P. Castagliola, A control chart for the multivariate coefficient of variation, Quality and Reliability Engineering International, vol. 32, pp. 1213-1225, 2016.

31. W.L. Teoh, J.K. Chong, M.B.C. Khoo, P. Castagliola, W.C. Yeong, Optimal designs of the variable sample size $\bar{x}$ chart based on median run length and expected median run length, Quality and Reliability Engineering International, vol. 33, pp. 121-134, 2017.

32. X.L. Hu, P. Castagliola, J.S. Sun, M.B.C. Khoo, The performance of variable sample size $\bar{x}$ chart with measurement errors, Quality and Reliability Engineering International, vol. 32, pp. 969-983, 2016.

33. X.Y. Chew, K.W. Khaw, W.C. Yeong, The efficiency of run rules schemes for the multivariate coefficient of variation: A Markov chain approach, Journal of Applied Statistics, vol. 47, pp. 460-480, 2020.

34. X.Y. Chew, M.B.C. Khoo, K.W. Khaw, W.C. Yeong, Z.L. Chong, A proposed variable parameter control chart for monitoring the multivariate coefficient of variation, Quality and Reliability Engineering International, vol. 35, pp. 2442-2461, 2019. 